\title{
Practical Synthesis of a S1P Receptor 1 Agonist via a
}

\section{Guareschi-Thorpe Reaction}

Gunther Schmidt, Martin H. Bolli, Cyrille Lescop, and Stefan Abele*

Chemistry Process R\&D, Actelion Pharmaceuticals Ltd., Gewerbestrasse 16, CH-4123 Allschwil,

Switzerland

* Corresponding author. E-Mail: stefan.abele@actelion.com. Telephone: +41 615656759.

Table of content:

1. Analytical methods

pages $\mathrm{S} 2-6$

2. Representative spectra or chromatograms:

$3\left({ }^{1} \mathrm{H}\right.$ NMR $)$

page $\mathrm{S} 7$

$4\left({ }^{1} \mathrm{H}\right.$ NMR $)$

page $\mathrm{S} 8$

5 ( ${ }^{1} \mathrm{H}$ NMR)

page $\mathrm{S} 9$

$5\left({ }^{13} \mathrm{C}\right.$ NMR $)$

page $\mathrm{S} 10$

13 ( ${ }^{1} \mathrm{H}$ NMR)

page $S 11$

16( ${ }^{1} \mathrm{H}$ NMR)

page $\mathrm{S} 12$

$17\left({ }^{1} \mathrm{H} \mathrm{NMR}\right)$

page $\mathrm{S} 13$

$18\left({ }^{1} \mathrm{H}\right.$ NMR, ${ }^{13} \mathrm{C}$ NMR $)$

pages $\mathrm{S} 14-15$

$23\left({ }^{1} \mathrm{H}\right.$ NMR)

page $S 16$

$24\left({ }^{1} \mathrm{H}\right.$ NMR)

page $\mathrm{S} 17$

$25\left({ }^{1} \mathrm{H}\right.$ NMR, ${ }^{13} \mathrm{C}$ NMR $)$

page $S$ 18-19

1 ( ${ }^{1} \mathrm{H}$ and ${ }^{13} \mathrm{C}$ NMR, HPLC, DSC)

pages $S$ 20-24 


\section{Analytical methods}

LC-MS method 1:

Agilent G1956B (MS, Ionisation: ESI+, APCI), Agilent G1312B Bin Pump, Agilent G1315C DAD, Agilent G1316B (thermostated column compartment), Agilent G1367C (auto sampler)

Injection volume: $\quad 2 \mu \mathrm{L}$

Column: $\quad$ Eclipse Plus $\mathrm{C} 18,1.8 \mu \mathrm{m}, 2.1 \times 50 \mathrm{~mm}$

Column flow: $\quad 1 \mathrm{~mL} / \mathrm{min}$

Eluent: $\quad$ Eluent A: Water, $0.08 \%$ TFA (trifluoroacetic acid)

Eluent B: Acetonitrile, 0.012\% TFA

Gradient:

$2.0 \min$

$95 \% \mathrm{~B}$

$2.8 \min$

$95 \% \mathrm{~B}$

$3.0 \mathrm{~min}$

$5 \% \mathrm{~B}$

Temperature: $\quad 50{ }^{\circ} \mathrm{C}$

Detection wavelength: $\quad 210 \mathrm{~nm}$ 


\section{LC-MS method 2:}

Agilent G1956B (MS, Ionisation: ESI+, APCI), Agilent G1312B Bin Pump, Agilent G1315C DAD, Agilent G1316B (thermostated column compartment), Agilent G1367C (auto sampler)

Injection volume: $\quad 2 \mu \mathrm{L}$

Column: $\quad$ Kinetex $\mathrm{C} 18,2.6 \mu \mathrm{m}, 2.1 \times 50 \mathrm{~mm}$

Column flow: $\quad 1 \mathrm{~mL} / \mathrm{min}$

Eluent: $\quad$ Eluent A: Water, $0.08 \%$ TFA (trifluoroacetic acid)

Eluent B: Acetonitrile, 0.012\% TFA

Gradient:

$2.0 \min$

$95 \%$ B

$2.8 \min$

$95 \% \mathrm{~B}$

$3.0 \mathrm{~min}$

$5 \% \mathrm{~B}$

Pressure: $\quad 370$ bar

Temperature: $\quad 40{ }^{\circ} \mathrm{C}$

Detection wavelength: $210 \mathrm{~nm}$ 


\section{GC-MS method:}

Thermo Trace GC Ultra, Thermo DSQ II MS detector, Thermo TriPlus Autosampler

Injection volume: $\quad 1 \mu \mathrm{L}$

Column: $\quad$ Zebron ZB-5 MS; $15 \mathrm{~m} \times 0.25 \mathrm{~mm} \mathrm{ID}, 0.25 \mathrm{~m}$ film

Column flow: $\quad 2 \mathrm{~mL} / \mathrm{min}$

Carrier gas: $\quad$ Helium

Split ratio: $\quad 20$

SSL inlet temp.: $\quad 200{ }^{\circ} \mathrm{C}$

Temp. gradient: $\quad 60-300{ }^{\circ} \mathrm{C}$ from 0 to $4.0 \mathrm{~min}, 300{ }^{\circ} \mathrm{C}$ isotherm from 4.0 to $5.0 \mathrm{~min}$

Ionization: $\quad$ Chemical ionization with $\mathrm{CH}_{4}$ as reagent gas 
HPLC method 1:

HPLC system Agilent 1100 with data acquisition (Chemstation Plus)

Injection volume: $\quad 5 \mu \mathrm{L}$

Column: $\quad$ Zorbax Eclipse XDB C18, $3.5 \mu \mathrm{m}, 150 \mathrm{~mm}$ x $4.6 \mathrm{~mm}$

Column flow: $\quad 1.0 \mathrm{~mL} / \mathrm{min}$

Eluent: $\quad$ Eluent A: water/MeOH/TFA (95/5/0.05)

Eluent B: water/MeOH/TFA (5/95/0.05)

$\begin{array}{lll}\text { Gradient mode: } & \text { Time } & \% \mathrm{~A} \\ & 0 \mathrm{~min} & 40 \% \\ & 1 \mathrm{~min} & 40 \% \\ & 7 \mathrm{~min} & 0 \% \\ & 22 \mathrm{~min} & 0 \% \\ & 22.1 \mathrm{~min} & 40 \% \\ & 27 \mathrm{~min} & 40 \% \\ \text { Pressure: } & 200 \mathrm{bar} & \\ \text { Temperature: } & 30{ }^{\circ} \mathrm{C} & \\ \text { Detection wavelength: } & 250 \mathrm{~nm} & \\ \text { Retention time of 1: } & 11.68 \mathrm{~min}\end{array}$


HPLC method 2:

HPLC system Agilent 1100 with data acquisition (Chemstation Plus)

Injection volume: $\quad 5 \mu \mathrm{L}$

Column: $\quad$ Daicel ChiralPak AD-H, $5 \mu \mathrm{m}, 250 \mathrm{~mm}$ x $4.6 \mathrm{~mm}$

Column flow: $\quad 0.8 \mathrm{~mL} / \mathrm{min}$

Eluent: $\quad$ Eluent A: Hexane/EtOH/diethylamine $(80 / 20 / 0.1)$

Eluent B: $\mathrm{EtOH}+0.1 \%$ diethylamine

Gradient mode: $\quad$ Gradient mode

Gradient mode: $\quad$ Time $\quad \% \mathrm{~A}$

$0 \min \quad 100 \%$

$15 \min \quad 100 \%$

$23 \min \quad 50 \%$

$26 \min \quad 100 \%$

$35 \min \quad 100 \%$

Pressure: $\quad 35$ bar

Temperature: $\quad 25^{\circ} \mathrm{C}$

Detection wavelength: $\quad 250 \mathrm{~nm}$

Retention time of $\mathbf{1}$ : $\quad 10.7 \mathrm{~min}$

$S$ enantiomer: $\quad 14.5 \mathrm{~min}$ 


\section{Representative spectra}

${ }^{1} \mathrm{H}$ NMR spectrum of methyl 2-chloro-6-methoxyisonicotinate 3 (in $\mathrm{CDCl}_{3}$ ).<smiles>COc1cc(C(C)=O)cc(Cl)n1</smiles>

TschanM cryst1\#1
proton_np_c1 CDC13 D: $\backslash \backslash$ schm1dig 33

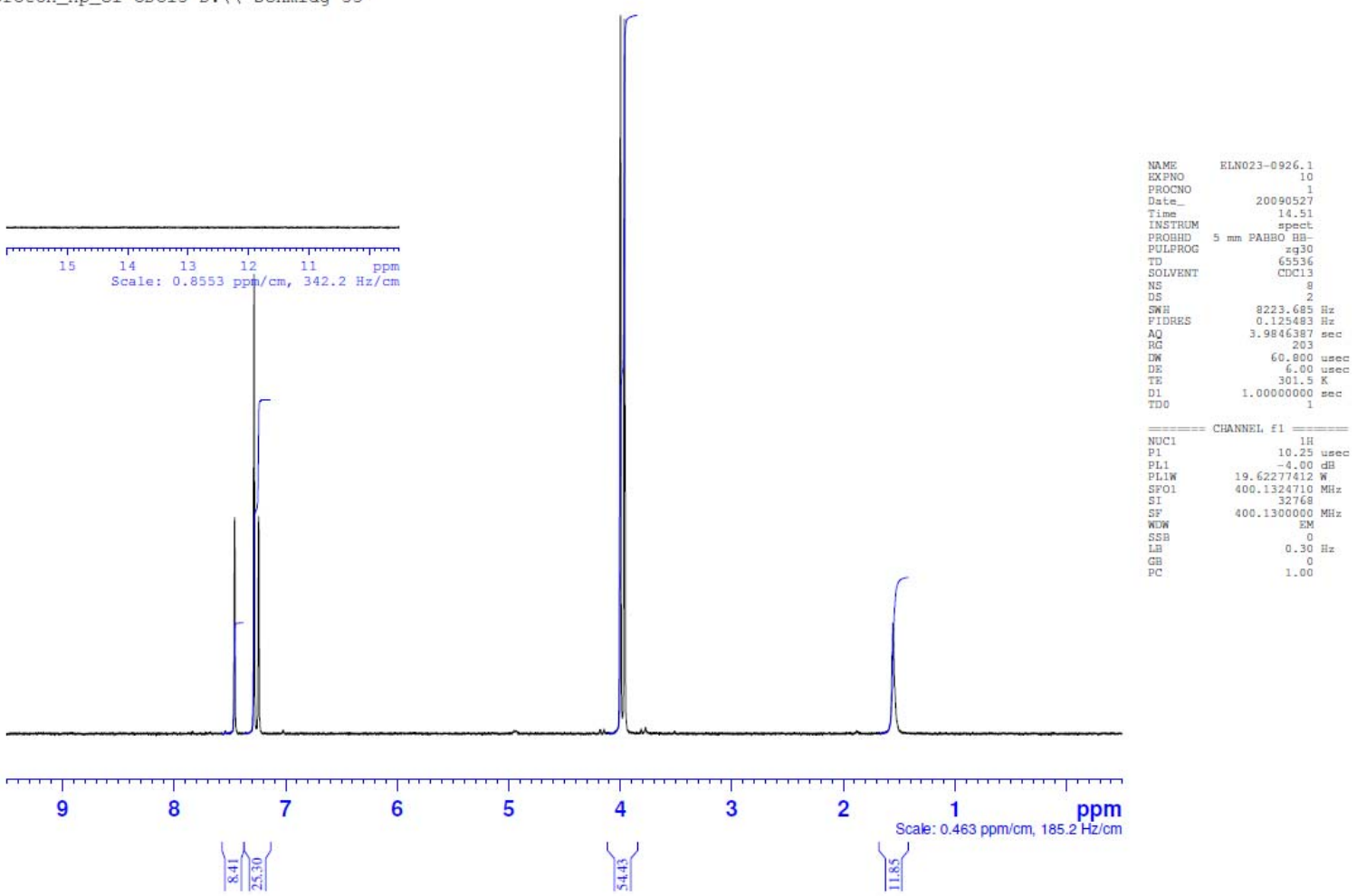


${ }^{1} \mathrm{H}$ NMR spectrum of methyl 2-cyclopentyl-6-methoxyisonicotinate 4 (in $\mathrm{CDCl}_{3}$ ).

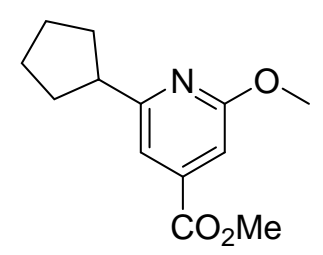

proton_np_cl CDC13 D: \\bollim 5

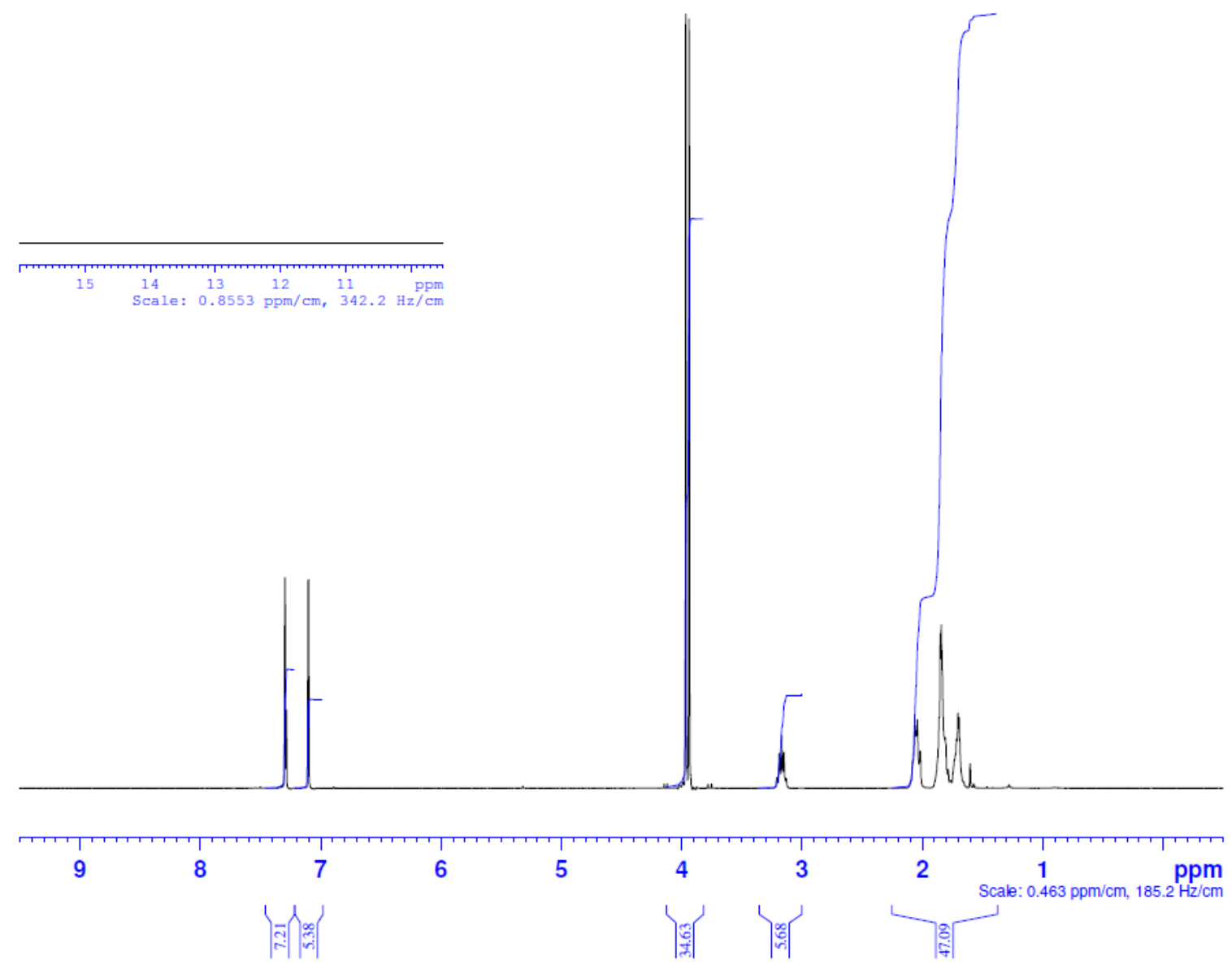


${ }^{1} \mathrm{H}$ NMR spectrum of 2-cyclopentyl-6-methoxyisonicotinic acid 5 (in $\mathrm{D}_{6}$ DMSO).<smiles>COc1cc(C(=O)O)cc(C2CCCC2)n1</smiles>

cryst $1 \# 1$

proton_np_c1 DMSO D: $\backslash \backslash$ schmidg 60
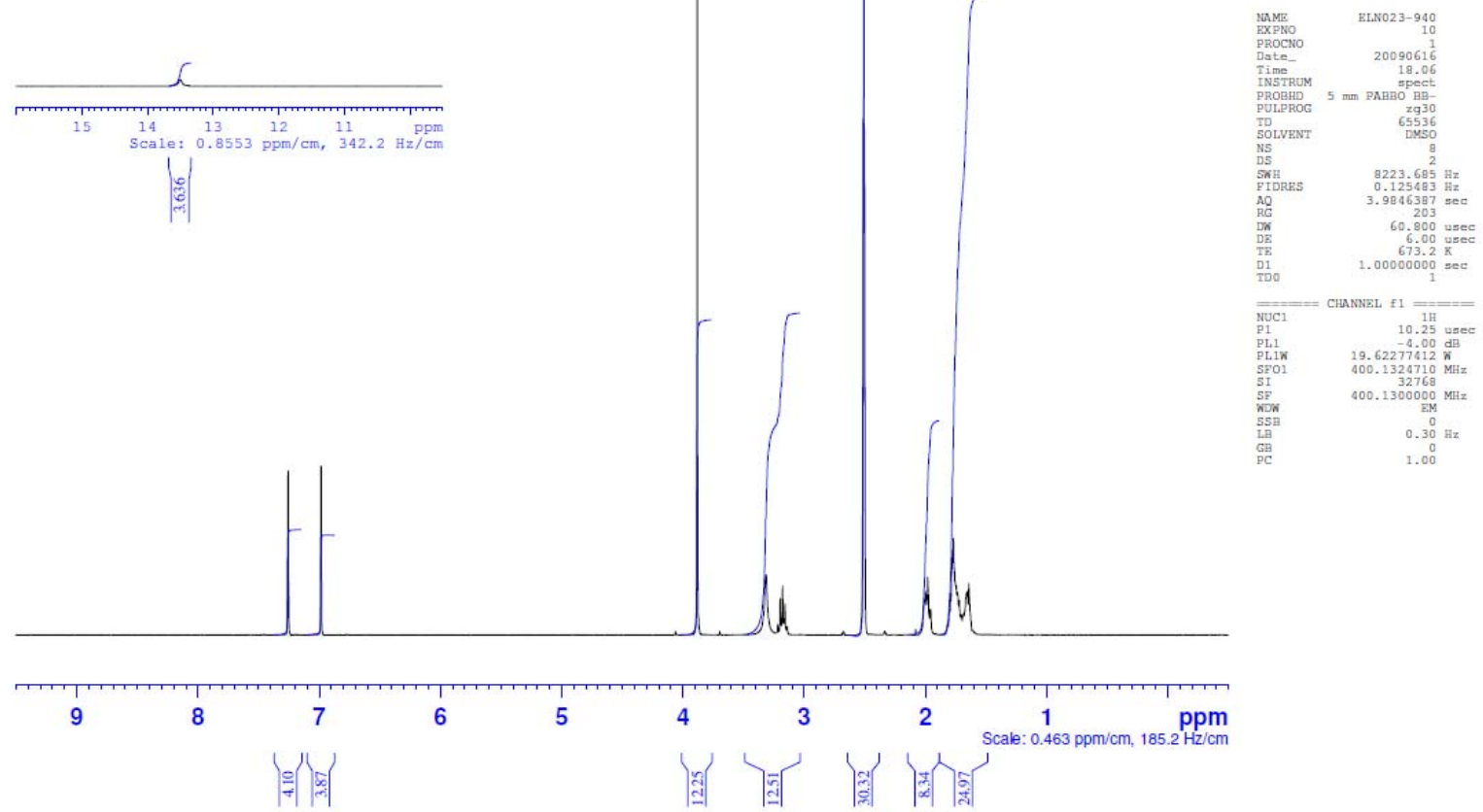
${ }^{13} \mathrm{C}$ NMR spectrum of 2-cyclopentyl-6-methoxyisonicotinic acid 5 (in $\mathrm{D}_{6} \mathrm{DMSO}$ ).

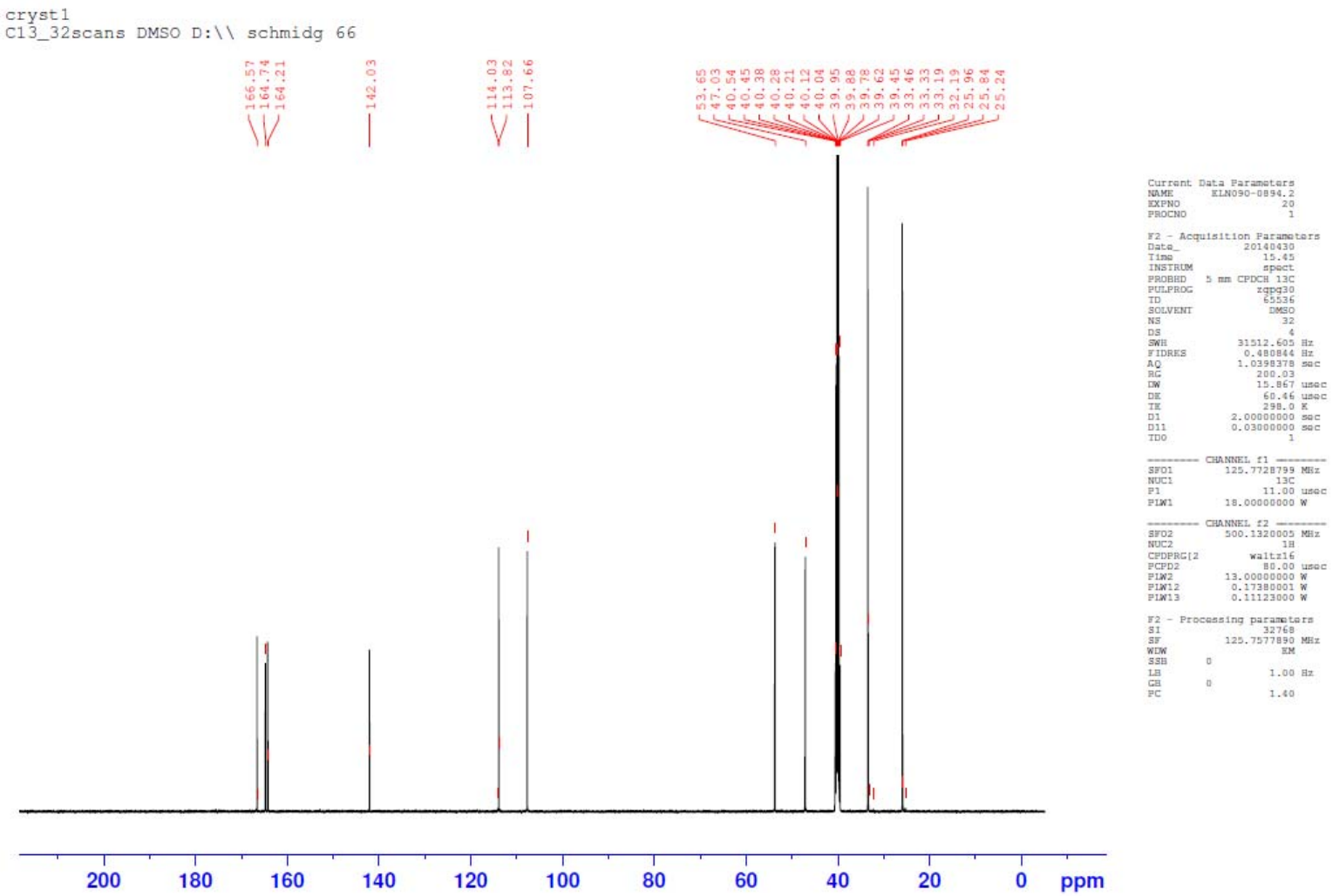


${ }^{1} \mathrm{H}$ NMR spectrum of 1-cyclopentylethan-1-one $\mathbf{1 3}$ (in $\mathrm{CDCl}_{3}$ ).<smiles>CC(=O)C1CCCC1</smiles>

dist1\#1

proton_np_c1 CDC13 D: $\backslash \backslash$ schmidg 4

O

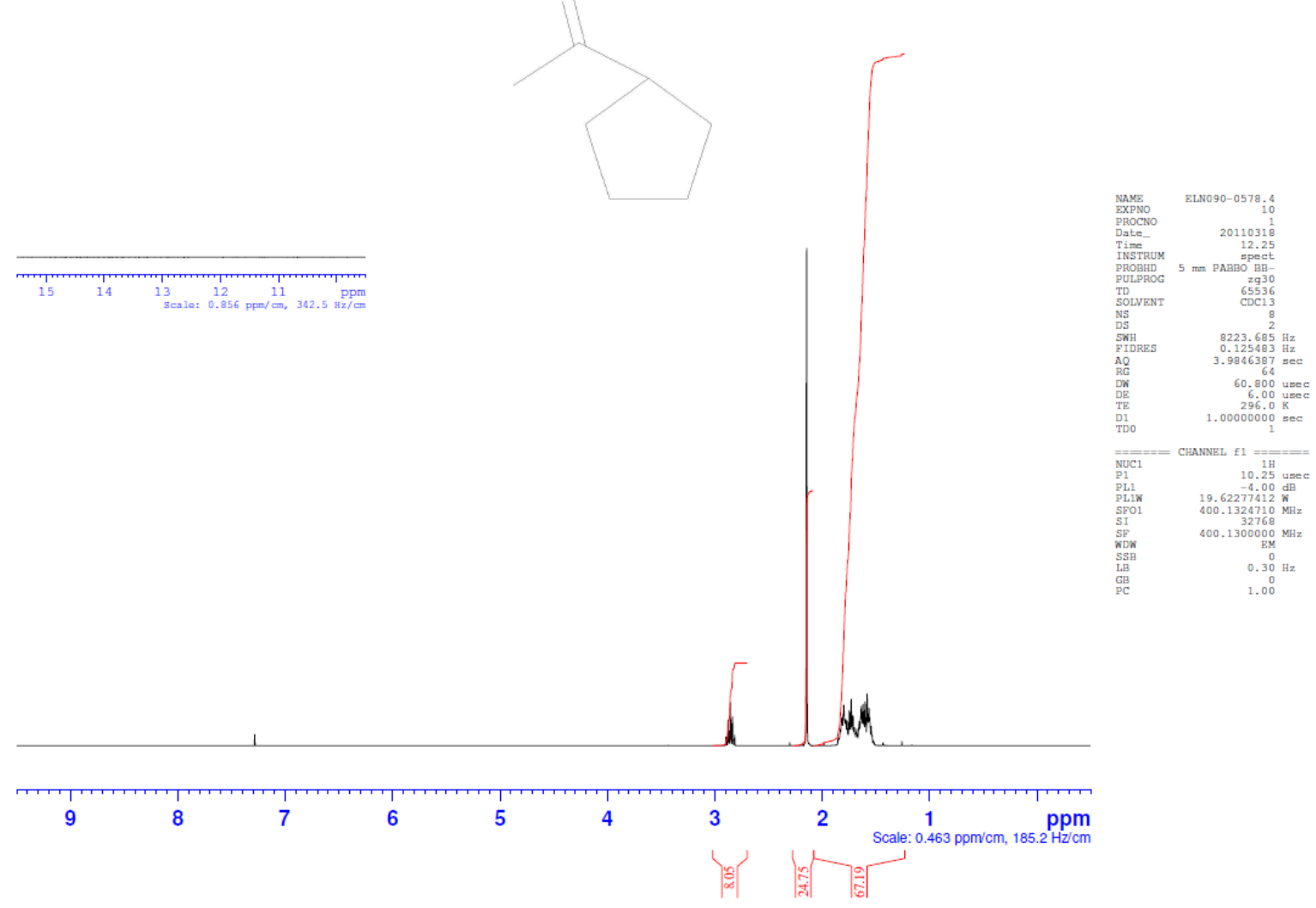

0 
${ }^{1} \mathrm{H}$ NMR spectrum of 2-cyclopentyl-6-hydroxyisonicotinic acid 16 (in $\mathrm{D}_{6} \mathrm{DMSO}$ ).<smiles>O=C(O)c1cc(C2CCCC2)[nH]c(=O)c1</smiles>

DoerrwP cryst1\#1

proton_np_Cl DMSO D: $\backslash \backslash$ schmidg 40

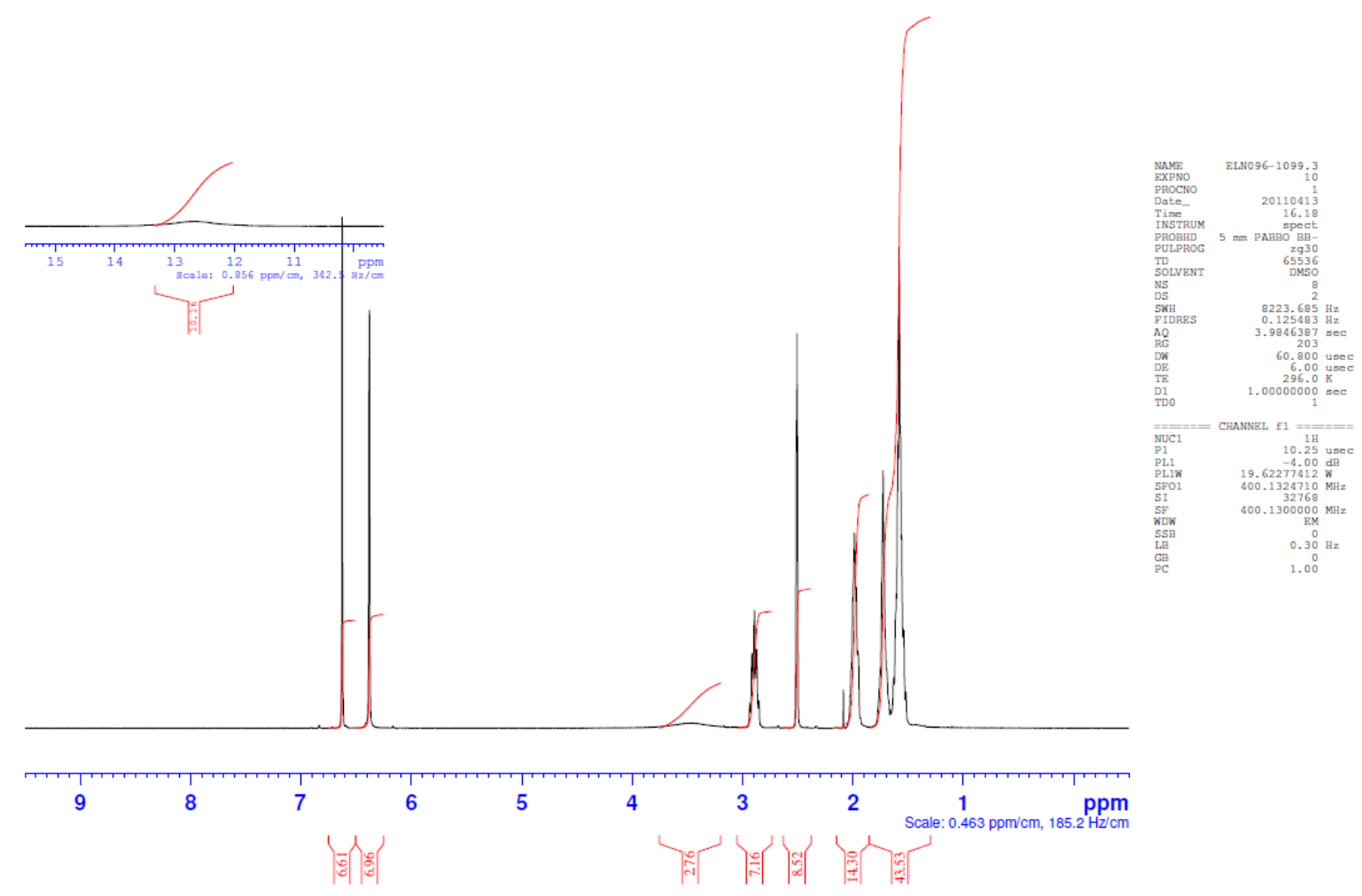


${ }^{1} \mathrm{H}$ NMR spectrum of methyl 2-cyclopentyl-6-hydroxyisonicotinate 17 (in $\mathrm{D}_{6} \mathrm{DMSO}$ ).<smiles>CC(=O)c1cc(C2CCCC2)[nH]c(=O)c1</smiles>

DoerrwP cryst1\#1
proton_np_cl DMSO D: $\backslash \backslash$ schmidg 40

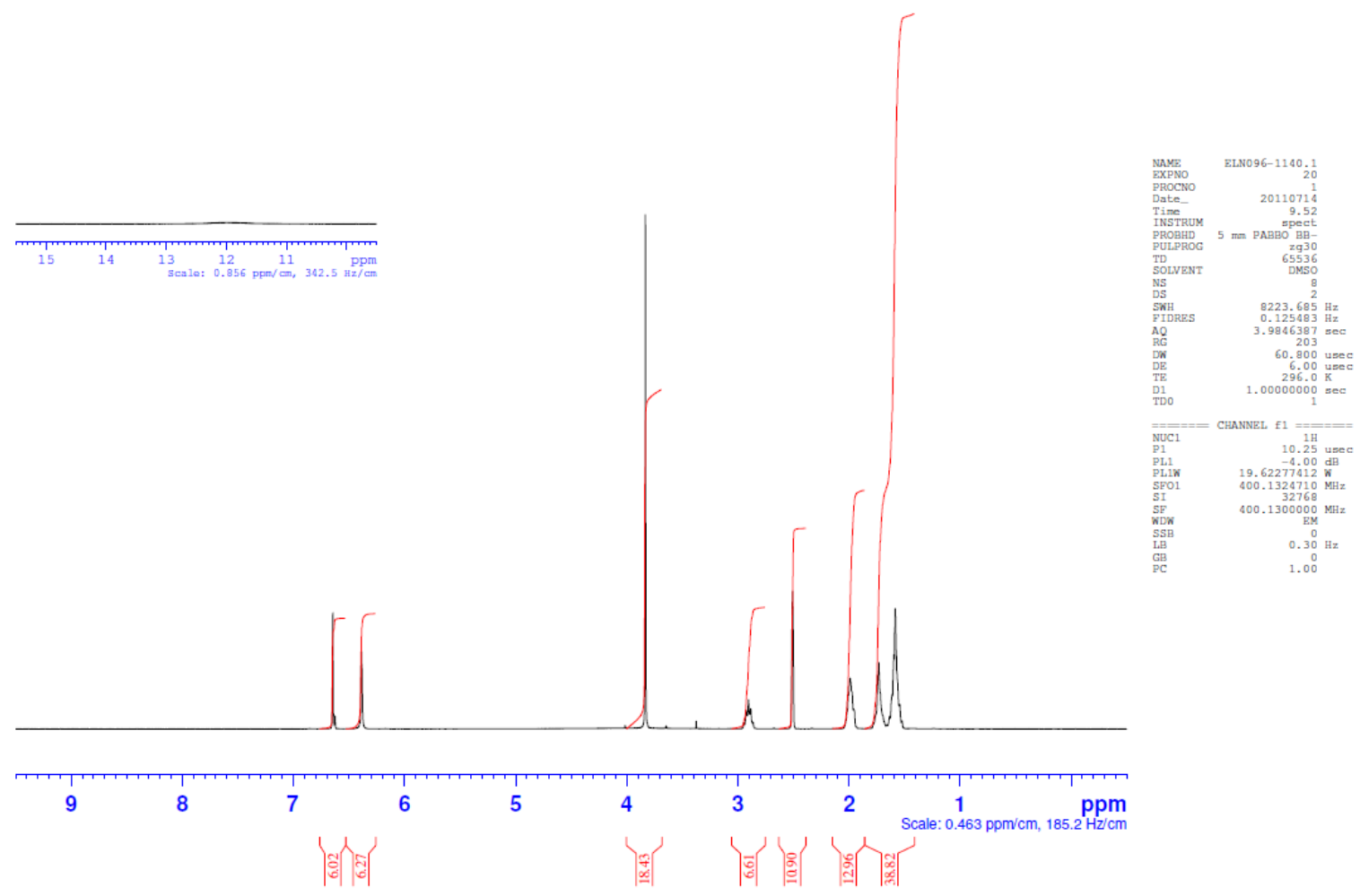


${ }^{1} \mathrm{H}$ NMR spectrum of methyl 2-chloro-6-cyclopentylisonicotinate 18 (in $\mathrm{CDCl}_{3}$ ).<smiles>CC(=O)c1cc(Cl)nc(C2CCCC2)c1</smiles>

DoerrwP crude1\#1

proton_np_cl CDC13 D: $\backslash \backslash$ schmidg 59

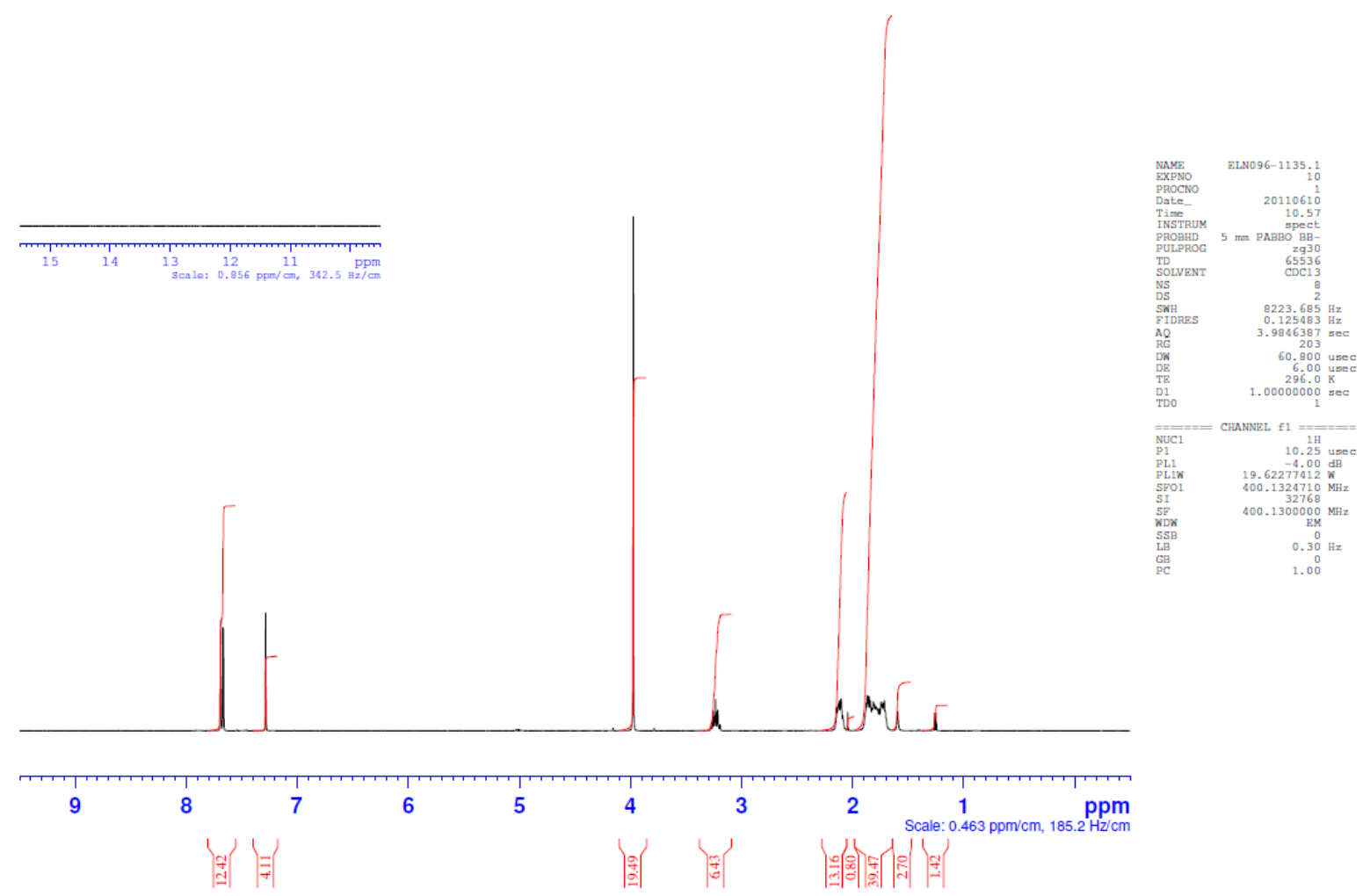


${ }^{13} \mathrm{C}$ NMR spectrum of methyl 2-chloro-6-cyclopentylisonicotinate 18 (in $\mathrm{CDCl}_{3}$ ).

crude $1 \#$

C13_32scans CDC13 D: $\backslash 1$ schmidg 90
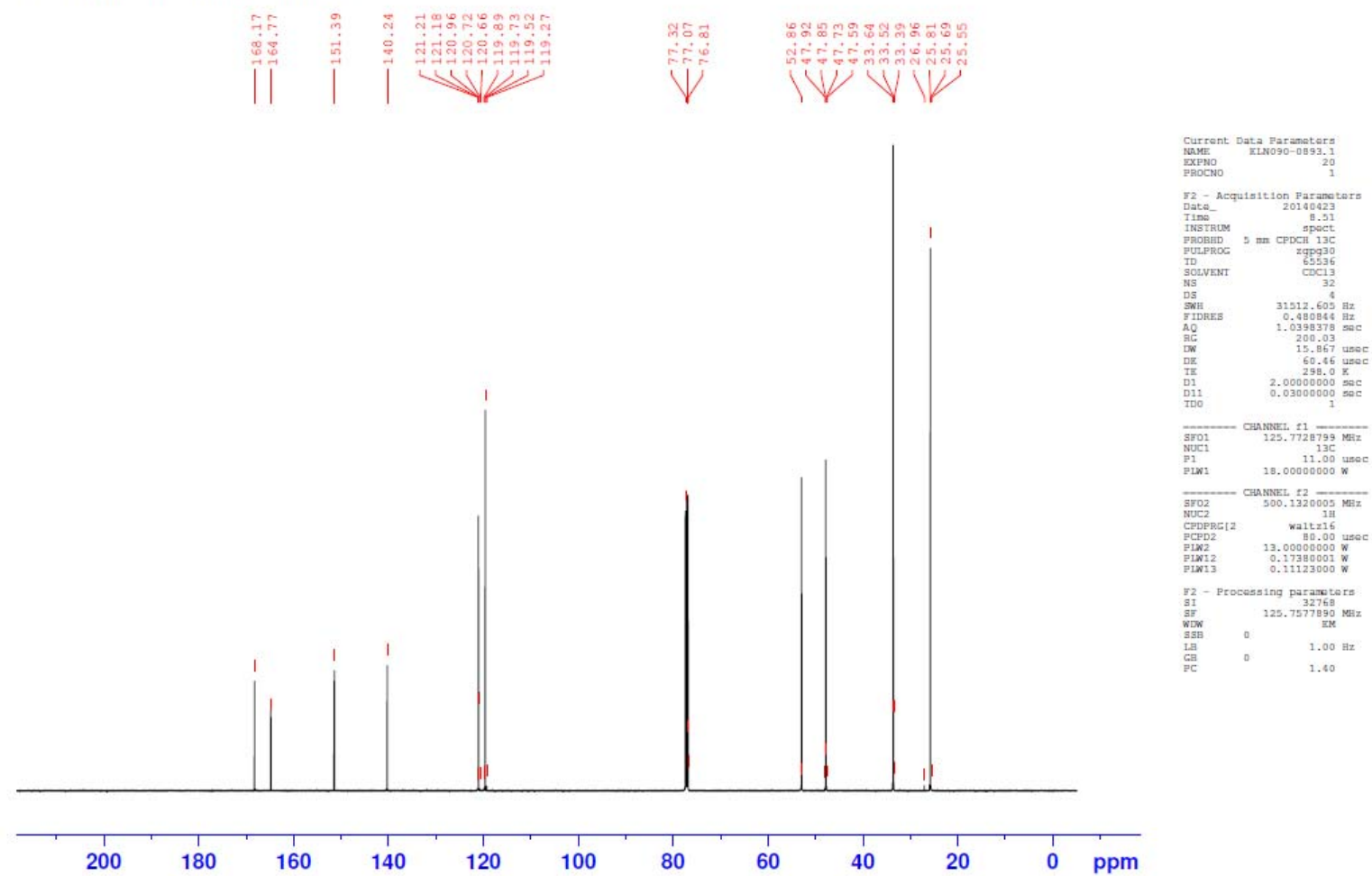
${ }^{1}$ H NMR spectrum of (R)-4-((2,2-dimethyl-1,3-dioxolan-4-yl)methoxy)-3-ethyl-N-hydroxy-5methylbenzimidamide 23 (in $\mathrm{CDCl}_{3}$ ).

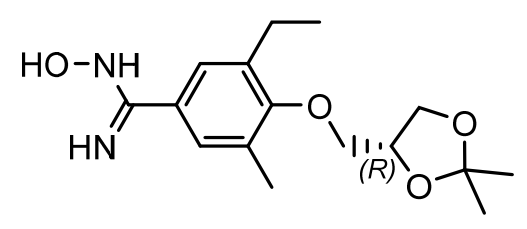

DoerrwP cryst1\#1
proton_np_c1 CDC13 D: $\backslash \backslash$ schm1dg 50

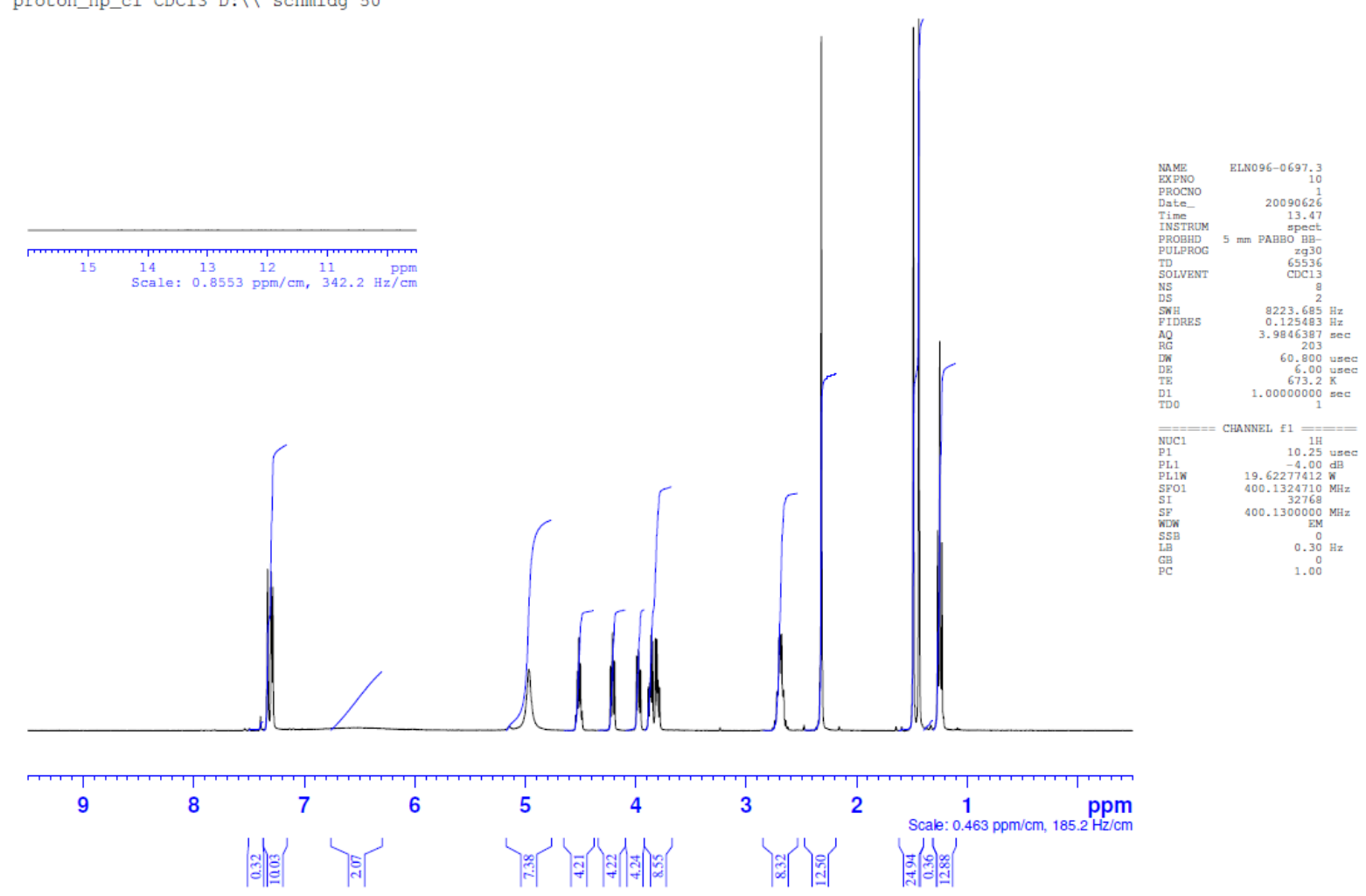


${ }^{1} \mathrm{H}$ NMR spectrum of (R)-N-((2-cyclopentyl-6-methoxyisonicotinoyl)oxy)-4-((2,2-dimethyl-1,3dioxolan-4-yl)methoxy)-3-ethyl-5-methylbenzimidamide 24 (in $\mathrm{CDCl}_{3}$ ).

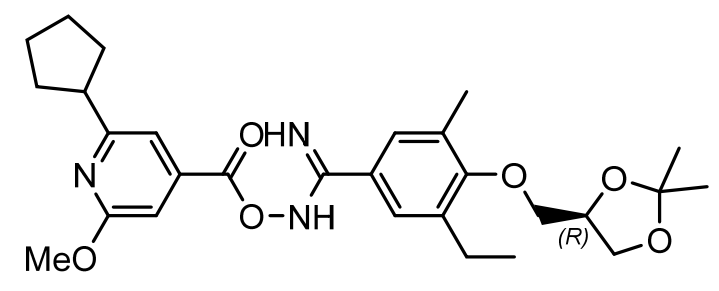

TschanM cryst1\#

proton_np_c1 CDC13 D: $\backslash \backslash$ schmidg 64

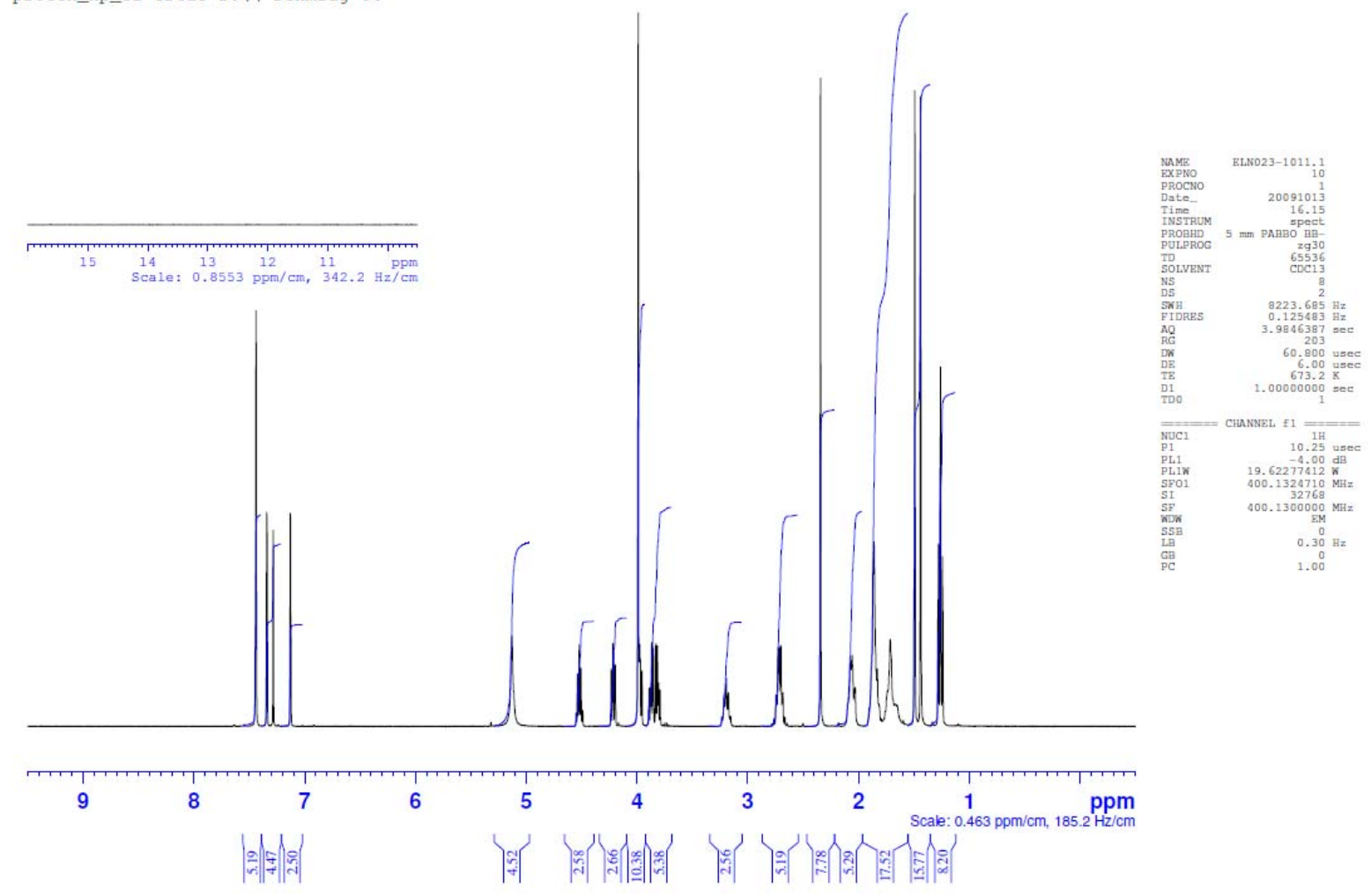


${ }^{1} \mathrm{H}$ NMR spectrum of (R)-5-(2-cyclopentyl-6-methoxypyridin-4-yl)-3-(4-((2,2-dimethyl-1,3-dioxolan4-yl)methoxy)-3-ethyl-5-methylphenyl)-1,2,4-oxadiazole 25 (in $\mathrm{CDCl}_{3}$ ).

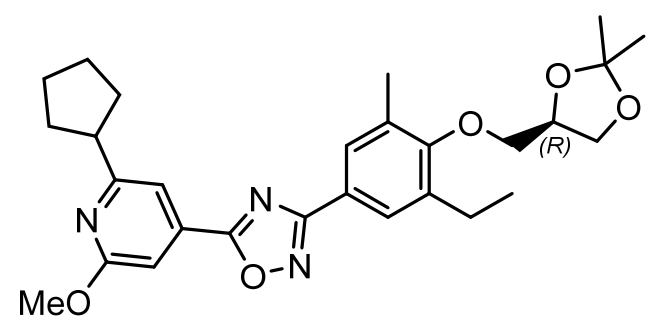

TschanM crude1\#1

proton_np_c1 CDC13 D: $\backslash \backslash$ schmidg 66
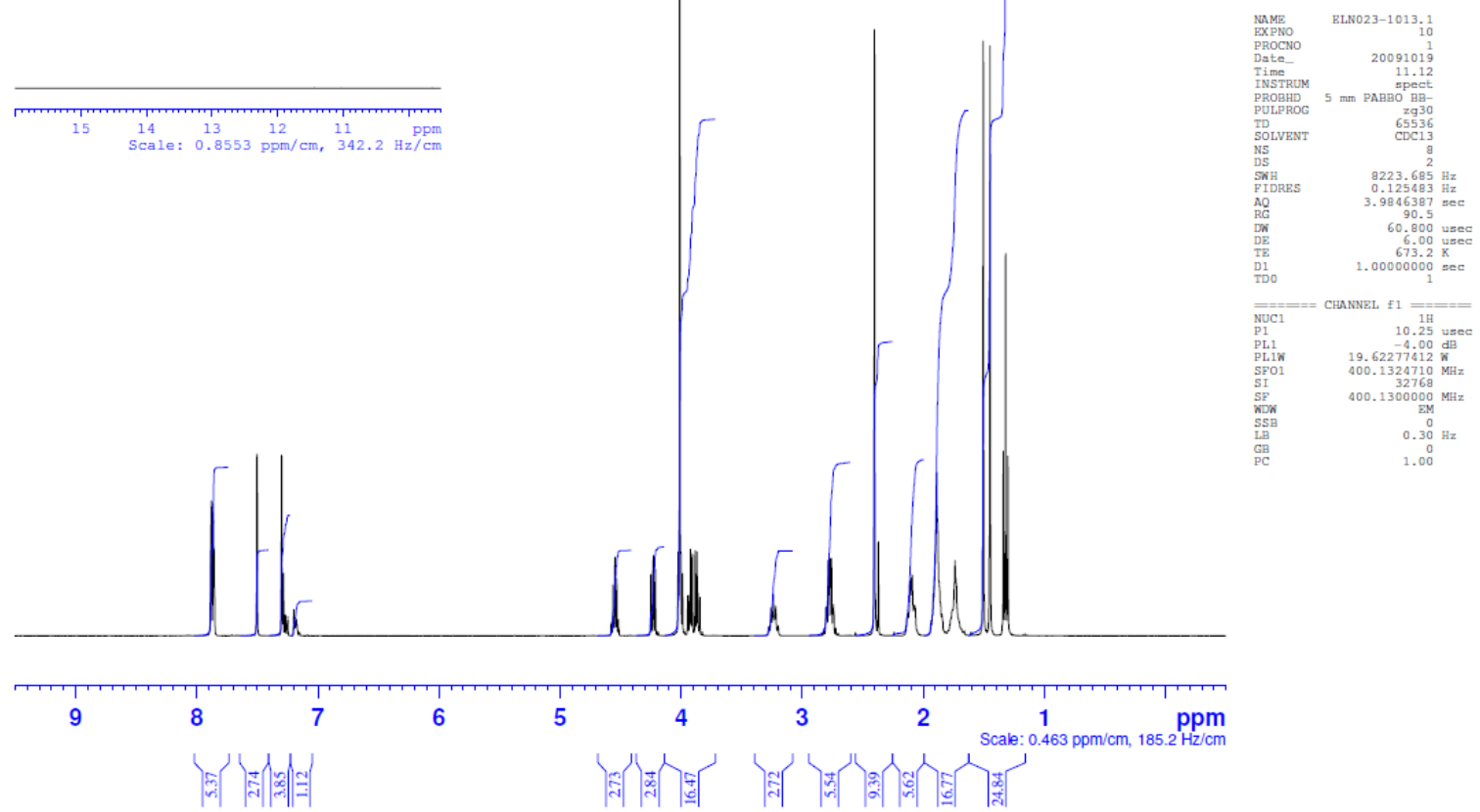
${ }^{13} \mathrm{C}$ NMR spectrum of (R)-5-(2-cyclopentyl-6-methoxypyridin-4-yl)-3-(4-((2,2-dimethyl-1,3dioxolan-4-yl)methoxy)-3-ethyl-5-methylphenyl)-1,2,4-oxadiazole 25 (in $\mathrm{CDCl}_{3}$ ).
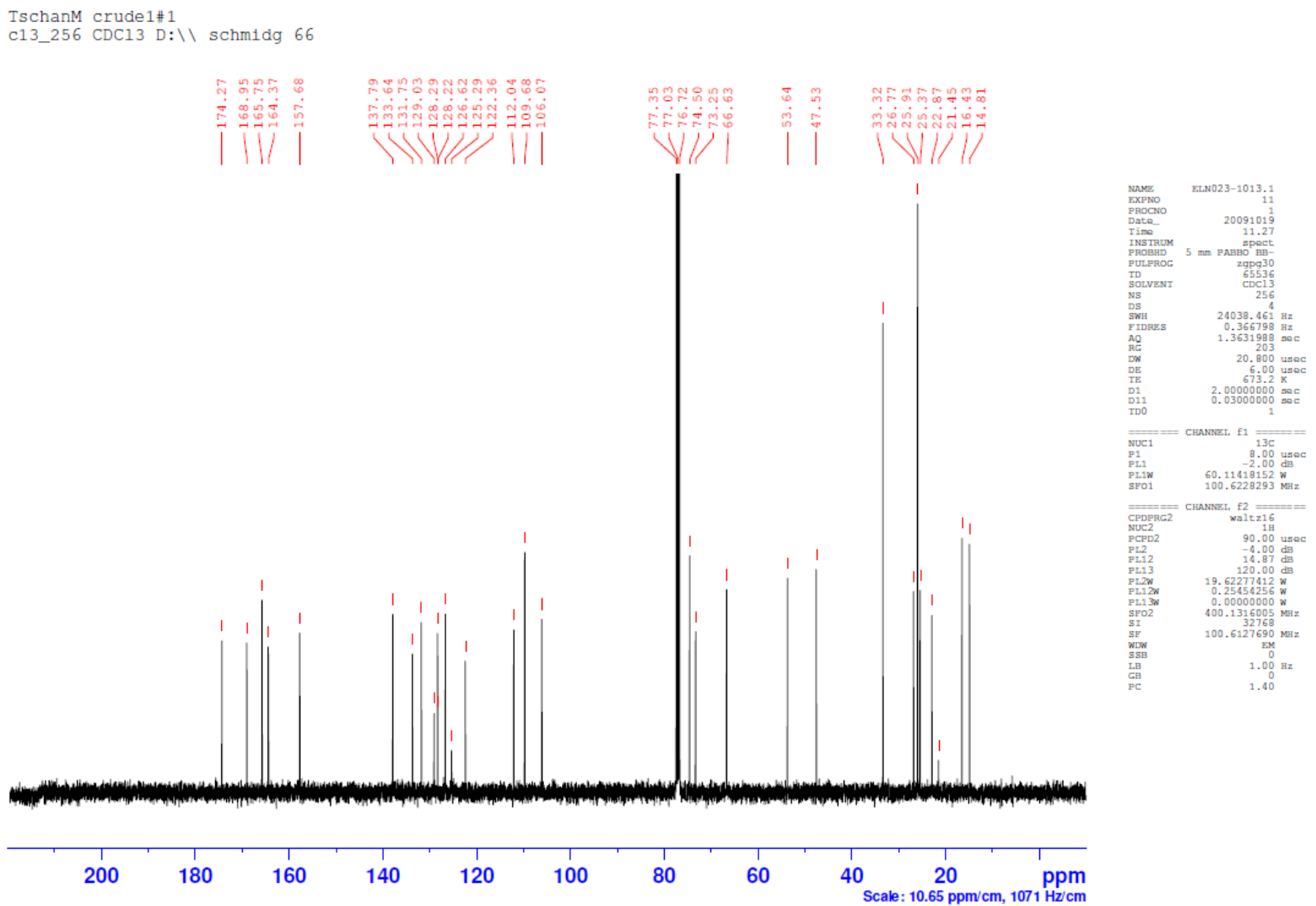
${ }^{1}$ H NMR spectrum of (S)-3-(4-(5-(2-cyclopentyl-6-methoxypyridin-4-yl)-1,2,4-oxadiazol-3-yl)-2ethyl-6-methylphenoxy)propane-1,2-diol 1 (in $\mathrm{D}_{6} \mathrm{DMSO}$ ).
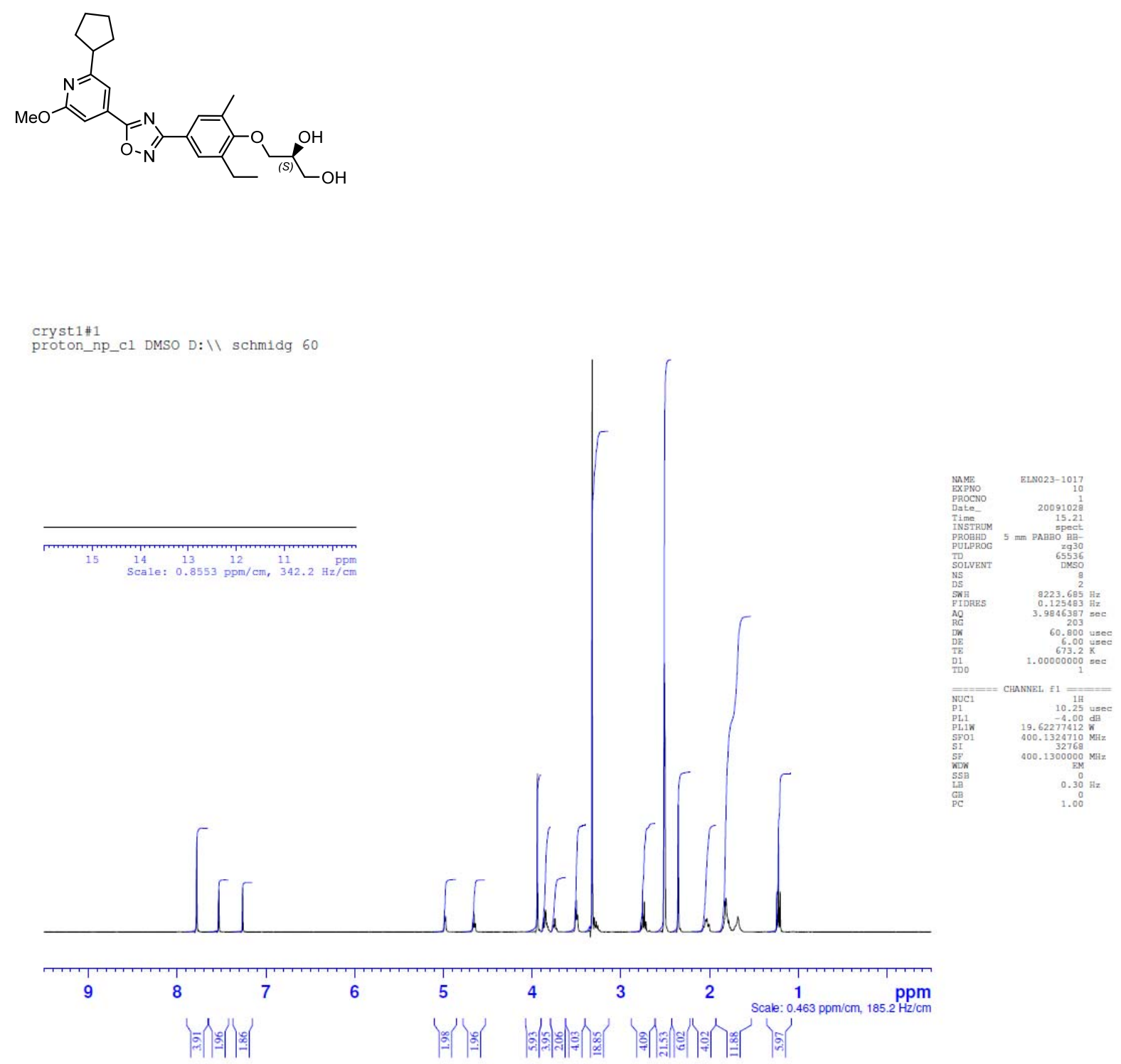
${ }^{13} \mathrm{C}$ NMR spectrum of (S)-3-(4-(5-(2-cyclopentyl-6-methoxypyridin-4-yl)-1,2,4-oxadiazol-3-yl)-2ethyl-6-methylphenoxy)propane-1,2-diol 1 (in $\mathrm{CDCl}_{3}$ ).

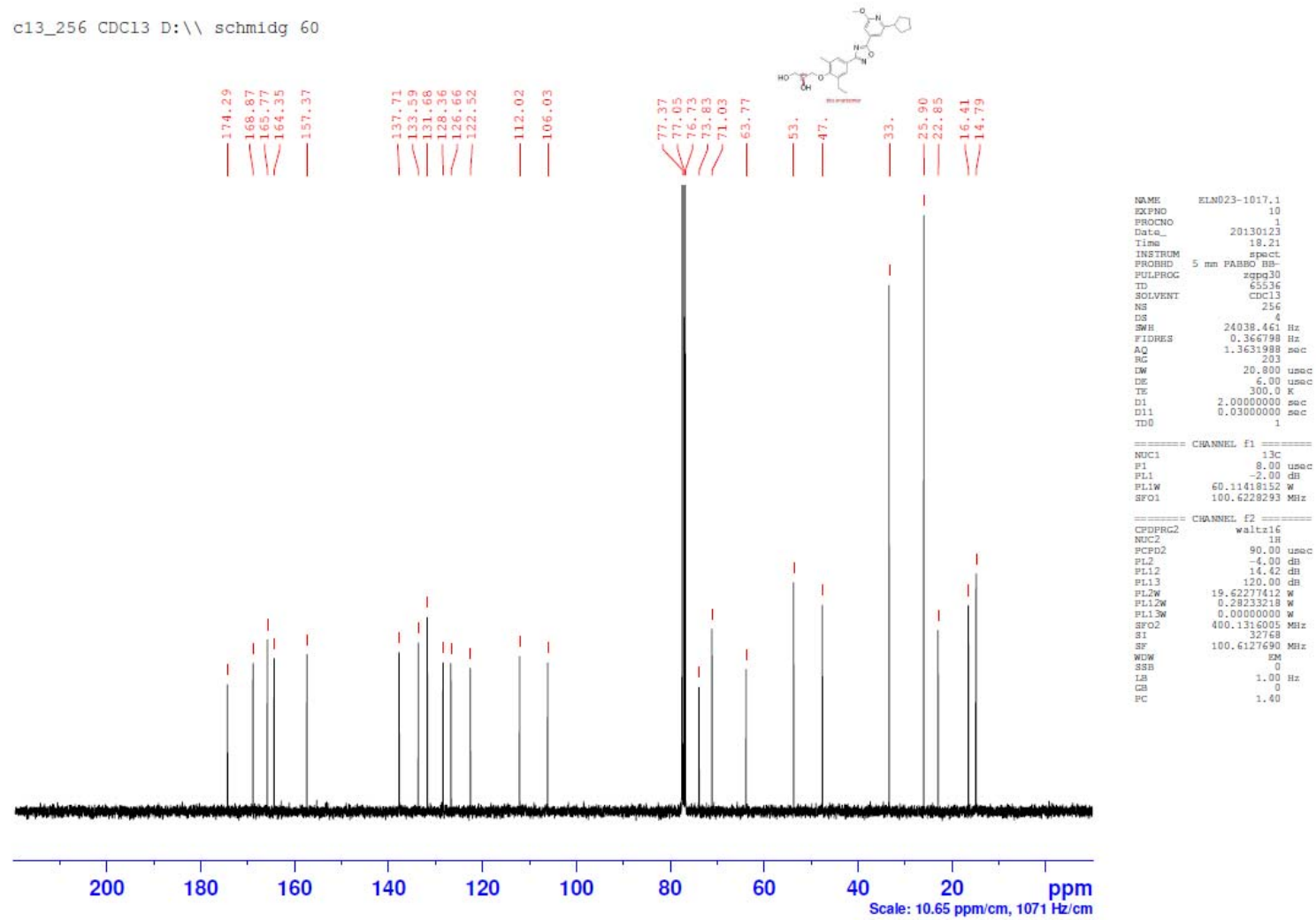


HPLC trace (HPLC method 1) of (S)-3-(4-(5-(2-cyclopentyl-6-methoxypyridin-4-yl)-1,2,4-oxadiazol3-yl)-2-ethyl-6-methylphenoxy)propane-1,2-diol 1

AM1010A

\begin{tabular}{llll|}
\hline \multicolumn{2}{c|}{ S A MPLE } & IN F O R MA T IO N \\
\hline Sample Name: & ElN023-1017.1 sample 1 & Acquired By: & denul \\
Sample Type: & Unknow n & Sample Set Name: DEL_091030_03A \\
Vial: & 2 & Acq. Method Set: & HPLC03_AM1010A_col2 \\
Injection \#: & 1 & Processing Method & AM1010A \\
Injection Volume: & 5.00 ul & Channel Name: & A1100 DAD AU Ch1 \\
Run Time: & 27.0 Mnutes & Proc. Chnl. Descr.: DAD AU Ch 1 Sample 250, Bw 4, \\
Date Acquired: & $10 / 31 / 2009$ 12:00:00 AM CET & & \\
Date Processed: & $11 / 2 / 2009$ 9:27:18 AM CET & & \\
\hline
\end{tabular}
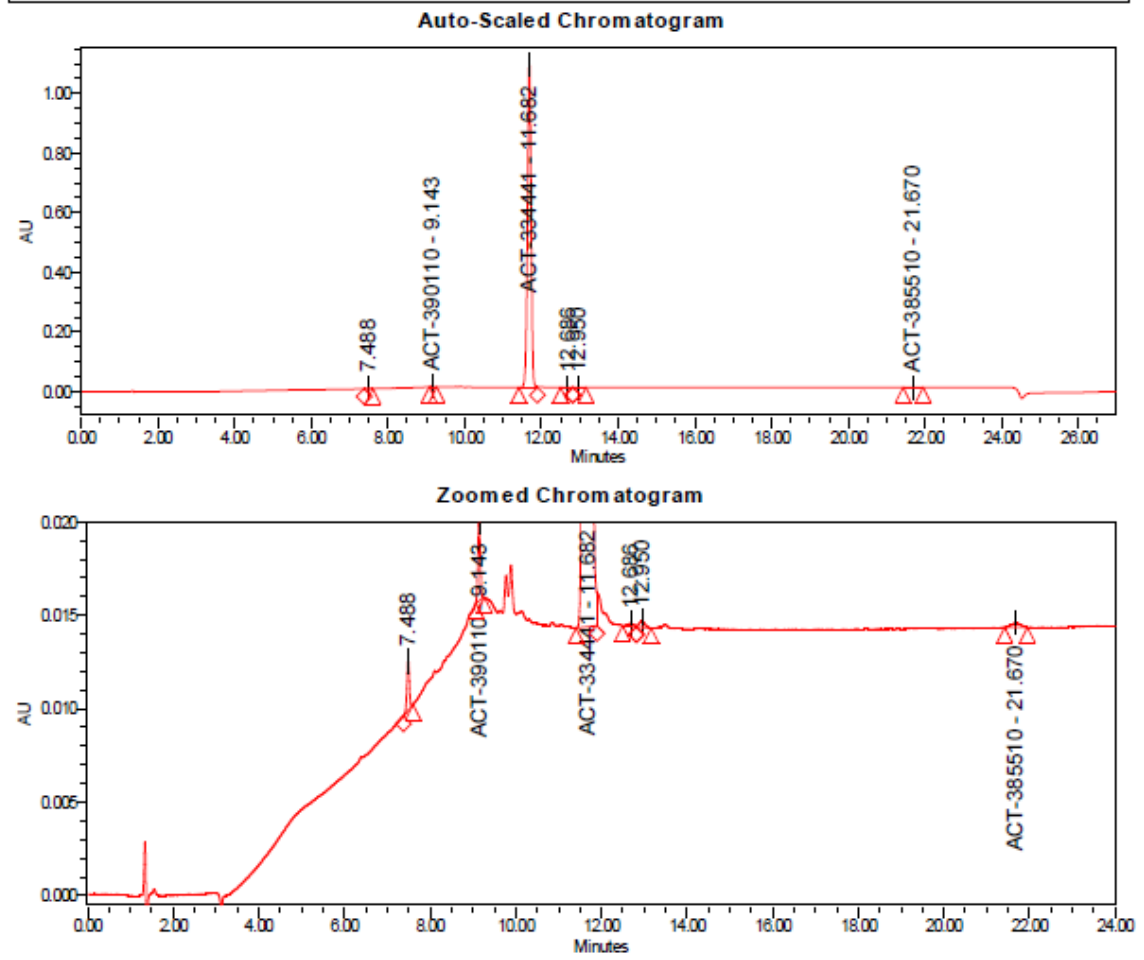

Reported by User: Laurent Denu (denul) Report Method: AM1010A

Report Method ID: 2834

Page: 1 of 4
Project Name: 2008IACT-334441IDSLab Date Printed:

$11 / 3 / 2009$ 3:55:18 PM Europe/Zurich

Peak Results

\begin{tabular}{|r|c|r|r|r|r|r|}
\hline & Name & RT Ratio & \multicolumn{1}{c|}{ RT } & Height & Area & $\%$ Area \\
\hline 1 & & 0.641 & 7.488 & 2635 & 10467 & 0.15 \\
\hline 2 & ACT-390110 & & 2.143 & 4227 & 16206 & 0.23 \\
\hline 3 & ACT-334441 & & 11.682 & 1086044 & 7080609 & 90.51 \\
\hline 4 & & 1.086 & 12.686 & 155 & 1629 & 0.02 \\
\hline 5 & & 1.100 & 12.860 & 353 & 3143 & 0.04 \\
\hline 6 & ACT-385510 & & 21.670 & 224 & 3425 & 0.05 \\
\hline
\end{tabular}


HPLC trace (HPLC method 2) of (S)-3-(4-(5-(2-cyclopentyl-6-methoxypyridin-4-yl)-1,2,4-oxadiazol3-yl)-2-ethyl-6-methylphenoxy)propane-1,2-diol 1

AM1012A

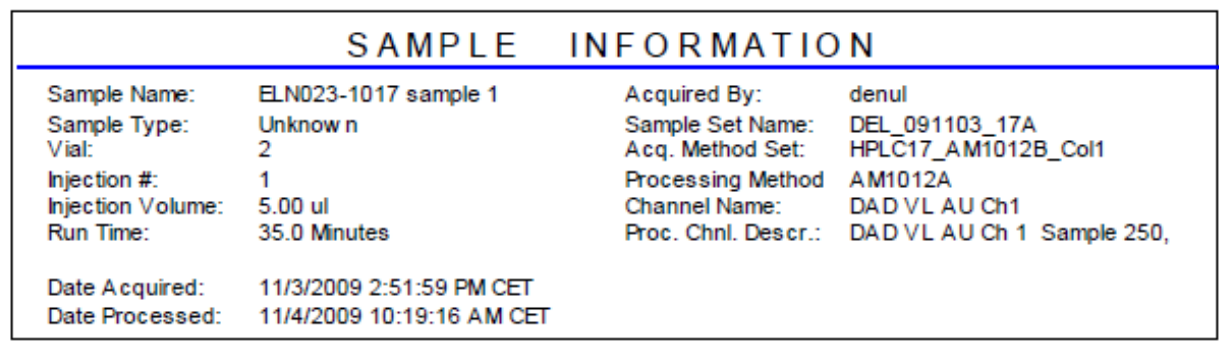

Auto-Scaled Chrom atogram

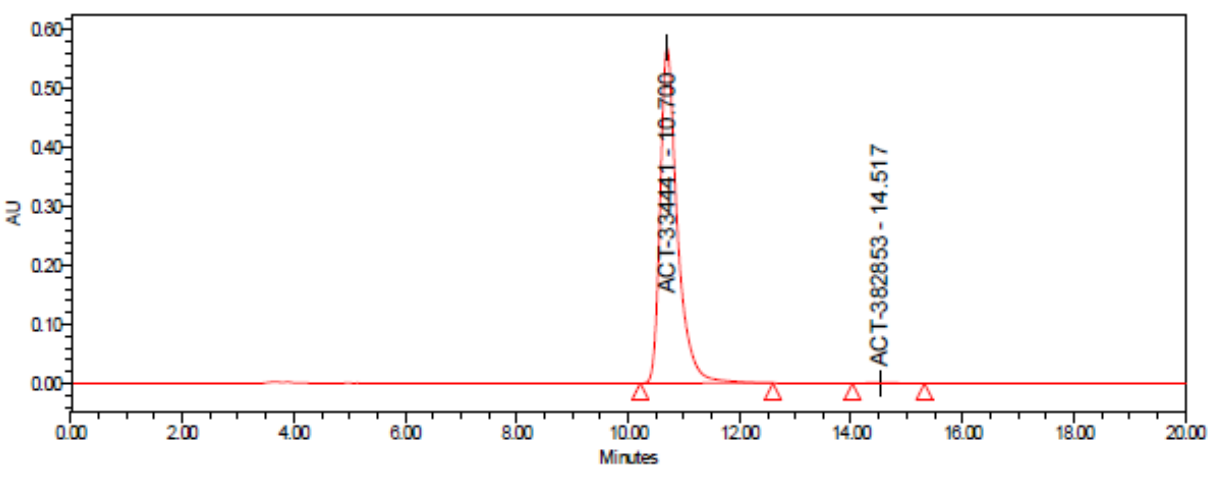

Zoomed Chrom atogram

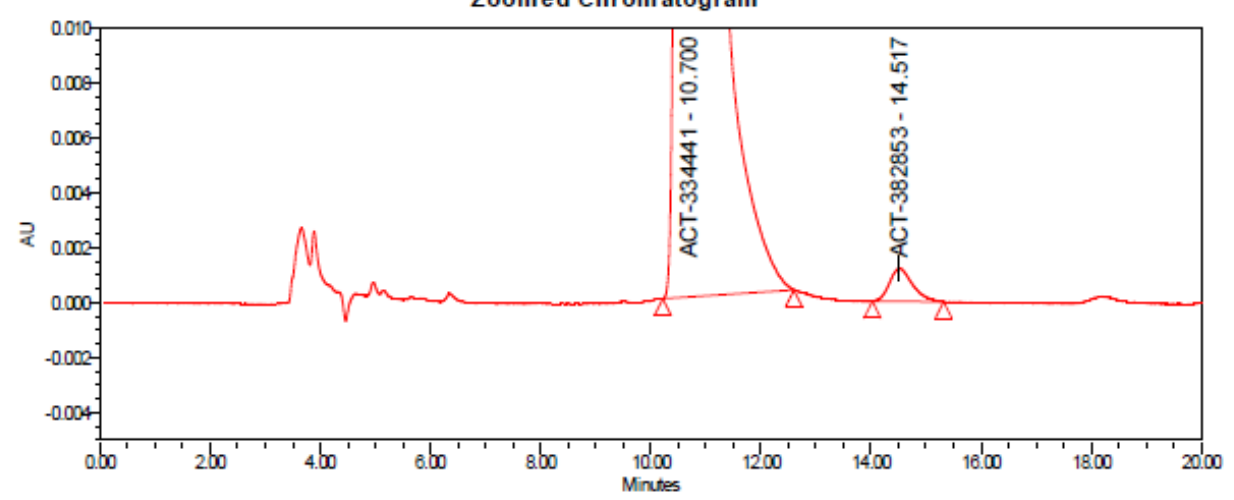

Reported by User: Laurent Denu (denul)

Project Name: 2008VACT-3344411DSLab

Report Method: AM1012A

Date Printed:

Report Method ID: 3118

$11 / 9 / 2009$

Page: 1 of 6

4:59:25 PM Europe/Zurich

Peak Results

\begin{tabular}{|c|c|c|r|r|r|}
\hline & Name & RT & \multicolumn{1}{c|}{ Area } & Height & $\%$ Area \\
\hline 1 & ACT-334441 & 10.700 & 12228150 & 508376 & 99.71 \\
\hline 2 & ACT-382953 & 14.517 & 35102 & 1212 & 0.28 \\
\hline
\end{tabular}


DSC of (S)-3-(4-(5-(2-cyclopentyl-6-methoxypyridin-4-yl)-1,2,4-oxadiazol-3-yl)-2-ethyl-6methylphenoxy)propane-1,2-diol 1

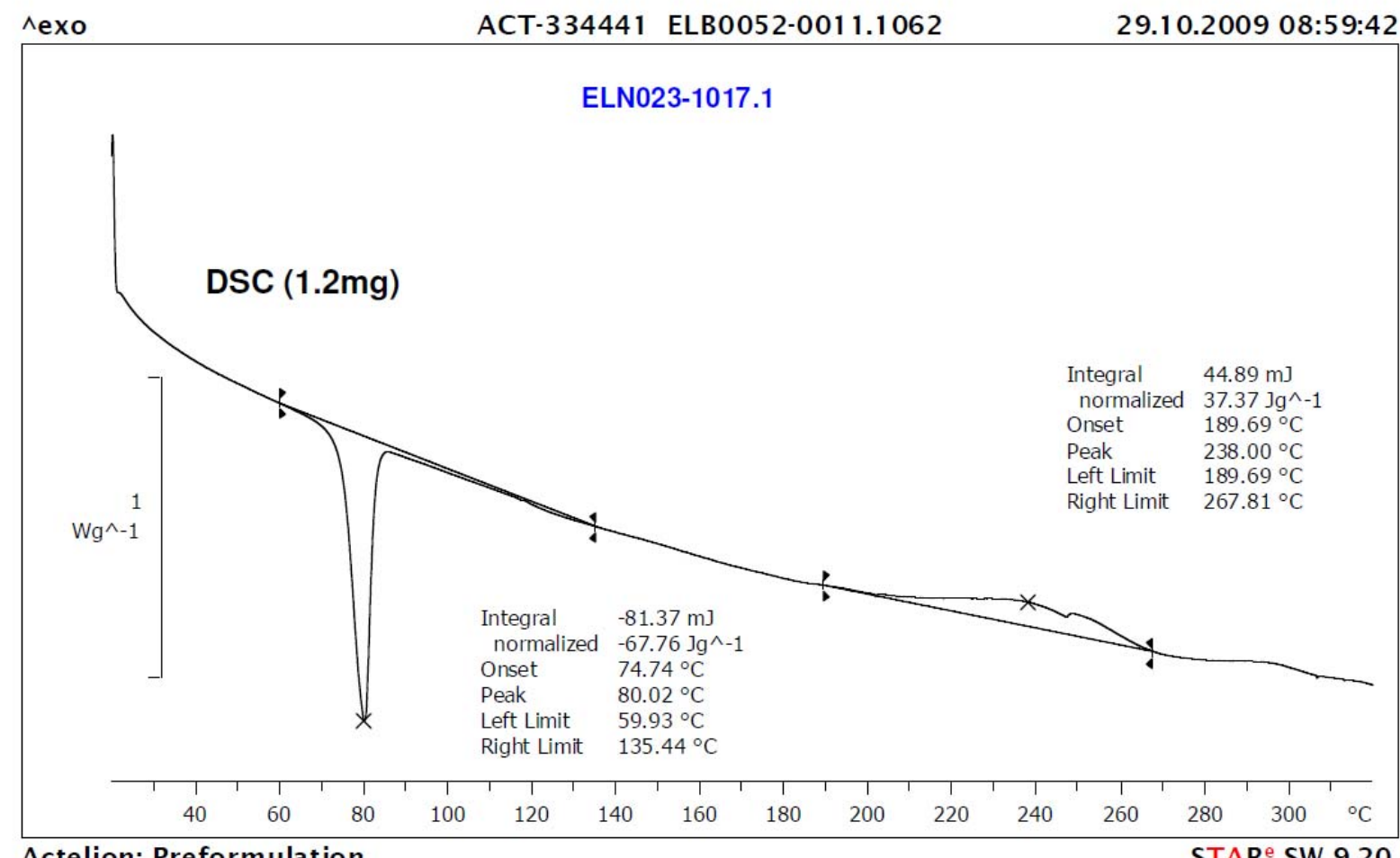

